\title{
Chemotactic and Other Responses of Plasmodia of Badhamia utricularis to an Extract of Stereum hirsutum and to Certain Other Substances
}

\author{
By D. KNOWLES* AND M. F. MADELIN \\ Department of Botany, The University, Bristol BS8 I $U G$
}

(Received 25 November 1974; revised 25 March 1975)

\begin{abstract}
SUMMARY
An extract of the basidiomycete Stereum hirsutum attracted plasmodia of Badhamia utricularis. Attracted plasmodia moved at a fairly constant speed until they contacted the source of attractant. The plasmodial front was directed towards the source by the production and advance of lobes at the nearest point of the front, and the attenuation and withdrawal of lobes in more remote parts. Directly applied extract halted the normal reversals of protoplasmic streaming in plasmodia and induced one-way flow for up to $25 \mathrm{~min}$. It also caused accumulation of protoplasm, and swelling and lengthening of the treated plasmodial strands. Benzamide, a non-volatile anaesthetic, also suppressed protoplasmic flow reversals and caused protoplasm to accumulate in swellings but did not cause chemotaxis. Extract from one other fungus, Metarrhizium anisopliae, possessed very similar activity to Stereum extract.
\end{abstract}

\section{INTRODUCTION}

Madelin, Audus \& Knowles (1975) reported that fruit bodies and mycelium of the basidiomycete Stereum hirsutum attracted plasmodia of the myxomycete Badhamia utricularis, and that the effective agent could be extracted in organic solvents and partially purified by phase separation and paper chromatography. The present paper describes the effects of extract of Stereum on plasmodia of Badhamia, which as well as positive chemotaxis include alterations in morphology and in rhythms of protoplasmic streaming, and compares them with the effects of extracts from five other fungi and benzamide.

\section{METHODS}

The sources of Badhamia utricularis and Stereum hirsutum, the methods of culturing them, and the procedures for making extracts are described by Madelin et al. (1975).

Metarrhizium anisopliae (Metch.) Sorok. (=Oospora destructor Metch.), Helminthosporium dematioideum Bubak \& Wroblenski, Epicoccum nigrum Link, Alternaria brassicicola (Schw.) Wiltshire, and an unnamed Penicillium from the culture collection of the Department of Botany, University of Bristol, were grown in Petri dishes on $2 \%$ malt extract agar at $25^{\circ} \mathrm{C}$. Extracts were prepared from plate cultures grown to a diameter of $4 \mathrm{~cm}$. The surface growth was scraped off the medium, immersed in $5 \mathrm{ml}$ acetone at room temperature for $30 \mathrm{~min}$ and removed by filtration through glass wool. The filtrates were phase-separated

* Present address: Department of Biochemistry, Imperial College of Science and Technology, London SW7 2BB. 
with equal volumes of petroleum ether (b.p. 40 to $60^{\circ} \mathrm{C}$ ), being inverted five times and left for $20 \mathrm{~min}$ before separation of the phases and retention of the acetone one. Aqueous solutions were prepared by evaporating a known volume of the acetone solution and redissolving the residue in the same volume of distilled water. The fractionation of extracts by paper chromatography with butanol-acetic acid-water, and the detection of positive chemotactic effectiveness in extracts by 'quadrant' and 'clock' tests were described by Madelin et al. (1975).

The migration of plasmodia was recorded either by drawing, at timed intervals, their outlines with a fine pen on the base of the enclosing Petri dish, or by time-lapse filming with a Paillard-Bolex I $6 \mathrm{~mm}$ cine camera which had a Som-Berthiot (Paris) Pan-cinor 85/2 telephoto lens with an auxiliary $\mathrm{I} \mathrm{m}$ close-up lens, Ilford $\mathrm{FP}_{4}$ film, and a timing device which illuminated the Petri dish containing the plasmodium for I s every $30 \mathrm{~s}$ and exposed one frame of the film every $60 \mathrm{~s}$ (i.e. during alternate light periods). Photomicrographs, including some in time-lapse sequence, were also taken by means of a Zeiss photomicroscope.

\section{RESULTS}

\section{The effects of Stereum extract on plasmodia of Badhamia}

Stereum extract and other test substances were either applied at a distance from plasmodia or applied directly to them. The results of these two sorts of treatment are presented separately.

Application at a distance from plasmodia. Experiments were done to determine how quickly plasmodia reacted to the application of Stereum extract several centimetres beyond the plasmodium margin, and how quickly plasmodia migrated when they had come under the influence of the attracting principle. In a series of experiments filter-paper discs, $5 \mathrm{~mm}$ in diameter, on which $0.2 \mathrm{ml}$ samples of Stereum fruit-body extract had been allowed to evaporate, were placed centrally in Petri dishes of plain agar on which small plasmodia were situated at various distances from the centre. The lids of the Petri dishes were sealed with petroleum jelly to avoid gradients of humidity or aeration. Migration of the plasmodia during incubation at room temperature $\left(18\right.$ to $20^{\circ} \mathrm{C}$ ) was recorded. In all the experiments essentially similar results were obtained. Most plasmodia commenced migration towards the discs within the first hour. Once a plasmodium commenced migration towards a disc it sustained a more or less constant centripetal velocity of around $3.5 \mathrm{~mm} / \mathrm{h}$ until it made contact. This is approximately the maximum speed shown in these tests by any plasmodium moving in the absence of attractant. However, in the absence of attractant the velocity was variable, plasmodia even spontaneously reversing their direction of movement.

The migration of an individual plasmodium was followed by means of time-lapse filming in order to observe details of its behaviour. The plasmodium was placed centrally upon a plate of $2 \%$ plain agar, and a filter-paper disc on to which $0.02 \mathrm{ml}$ of Stereum fruit body extract had been dried was placed near the edge of the plate which was then incubated at room temperature. A selection of the photographic results is presented in Fig. I. During the first few hours the plasmodium constantly extended and retracted processes or lobes in an apparently random way. After $6 \mathrm{~h} 40 \mathrm{~min}$ the lobe nearest the attractant disc branched, one branch advancing toward the disc and the other at right angles to this. The lobes thickened as protoplasm accumulated, and each branched into a series of smaller lobes which by $8 \mathrm{~h} 40 \mathrm{~min}$ amalgamated to form a large leading fan of plasmodium. At first this was not aimed directly toward the disc. However, the formation of new lobes on the part nearest the disc and withdrawal of those furthest from it resulted in the orientation of the 

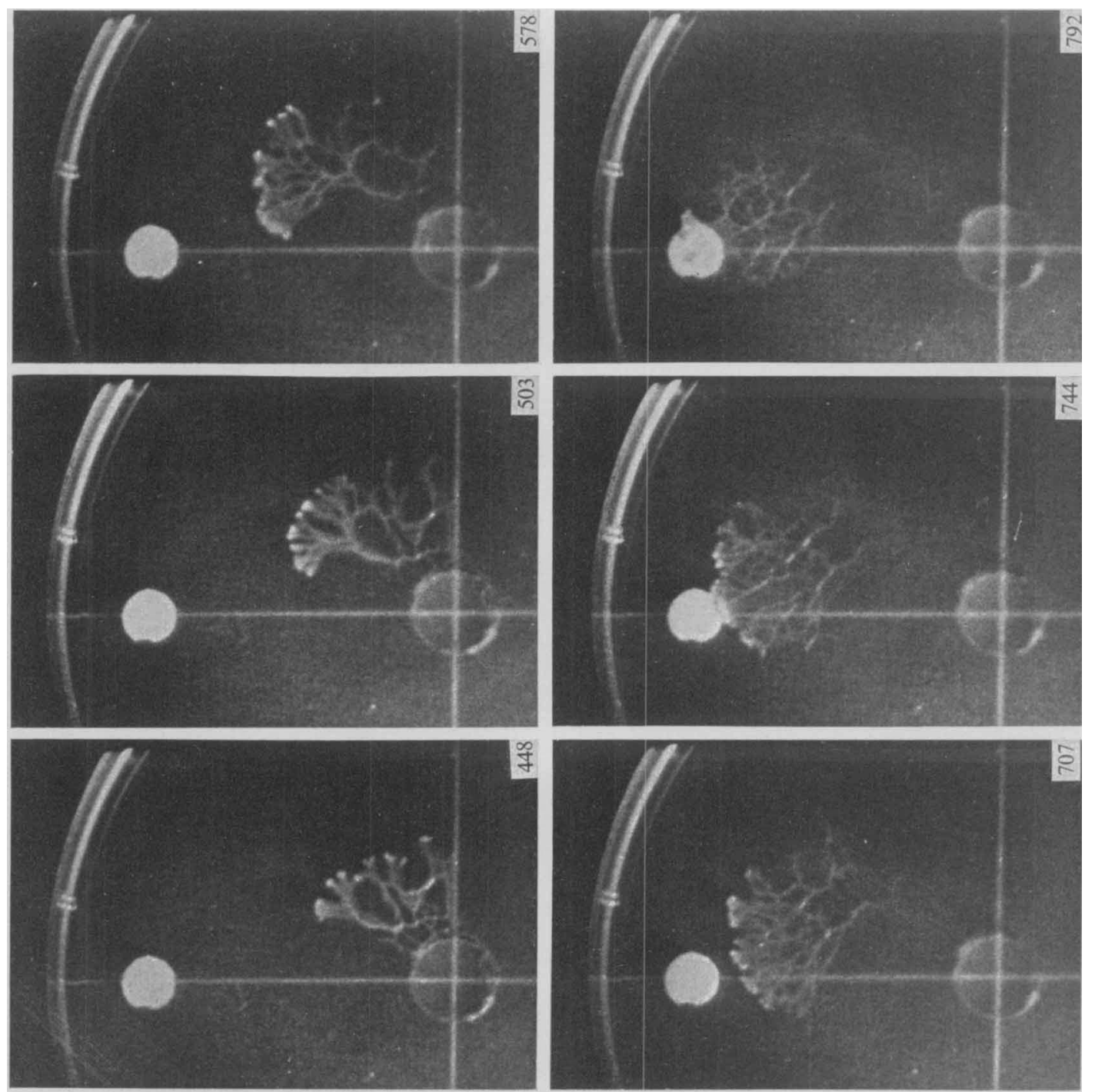

跑

홍

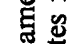

:

믕

a..

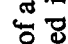

证

䆑.

8.

$\Phi$

苛

品

号

氜

要

สี

ธี 른

跤

额

쿵

居

匀

डै

놇

s.

$4:$

릴

;

密

类

$\pi$

प०

E

$\square$

등

a

号焉

옹
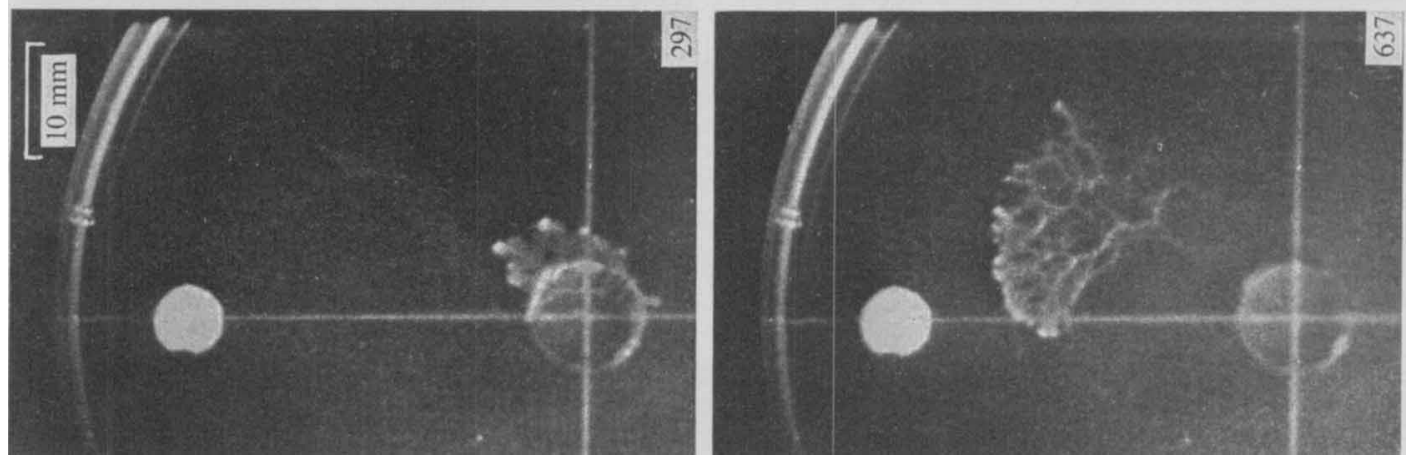

E⿱

\%

E

온

몽

돈

蛋

용

도

惢

ㄴㅇㅇ

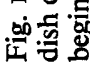


D. KNOWLES AND M. F. MADELIN
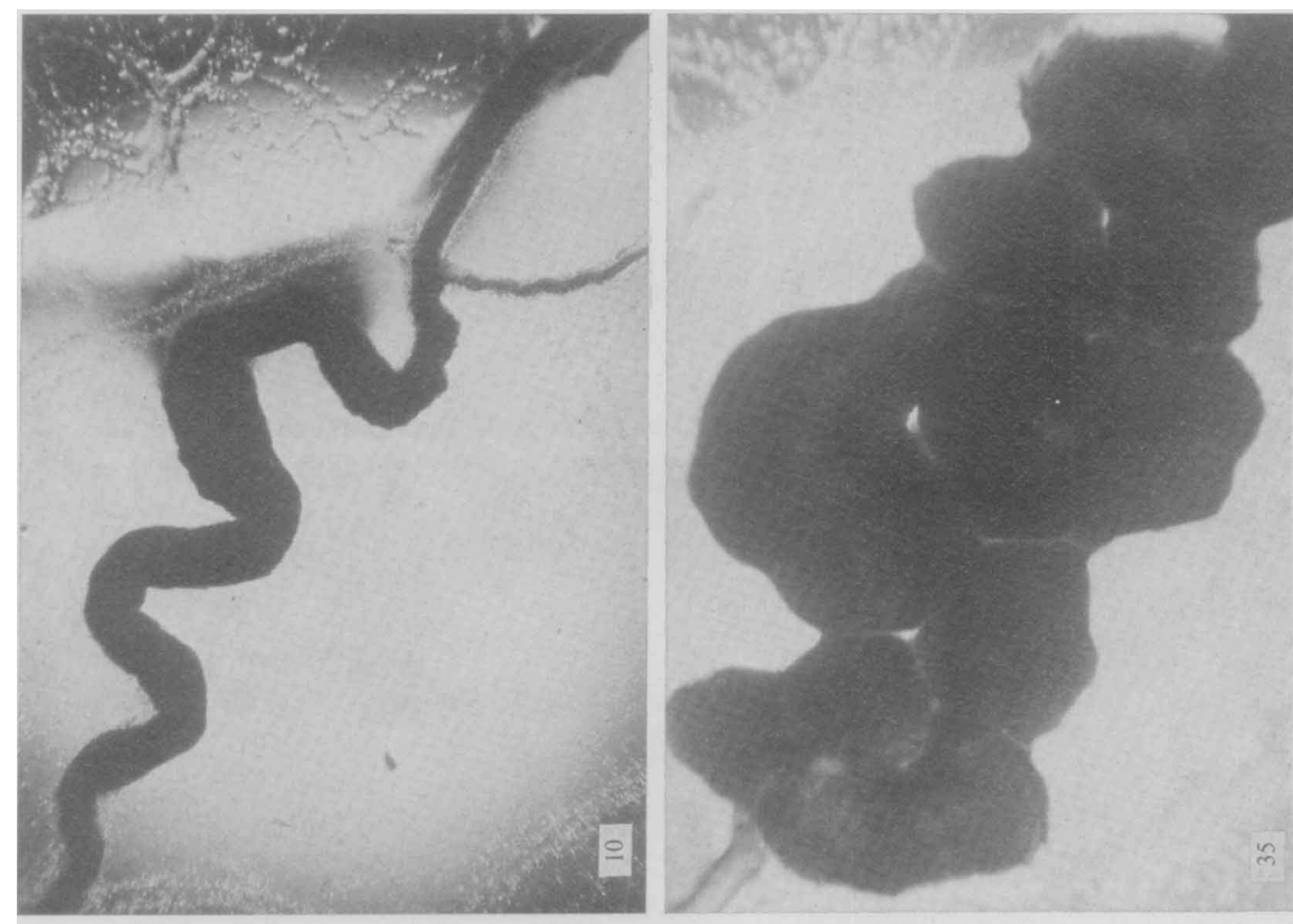

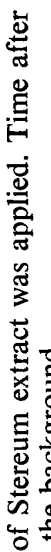
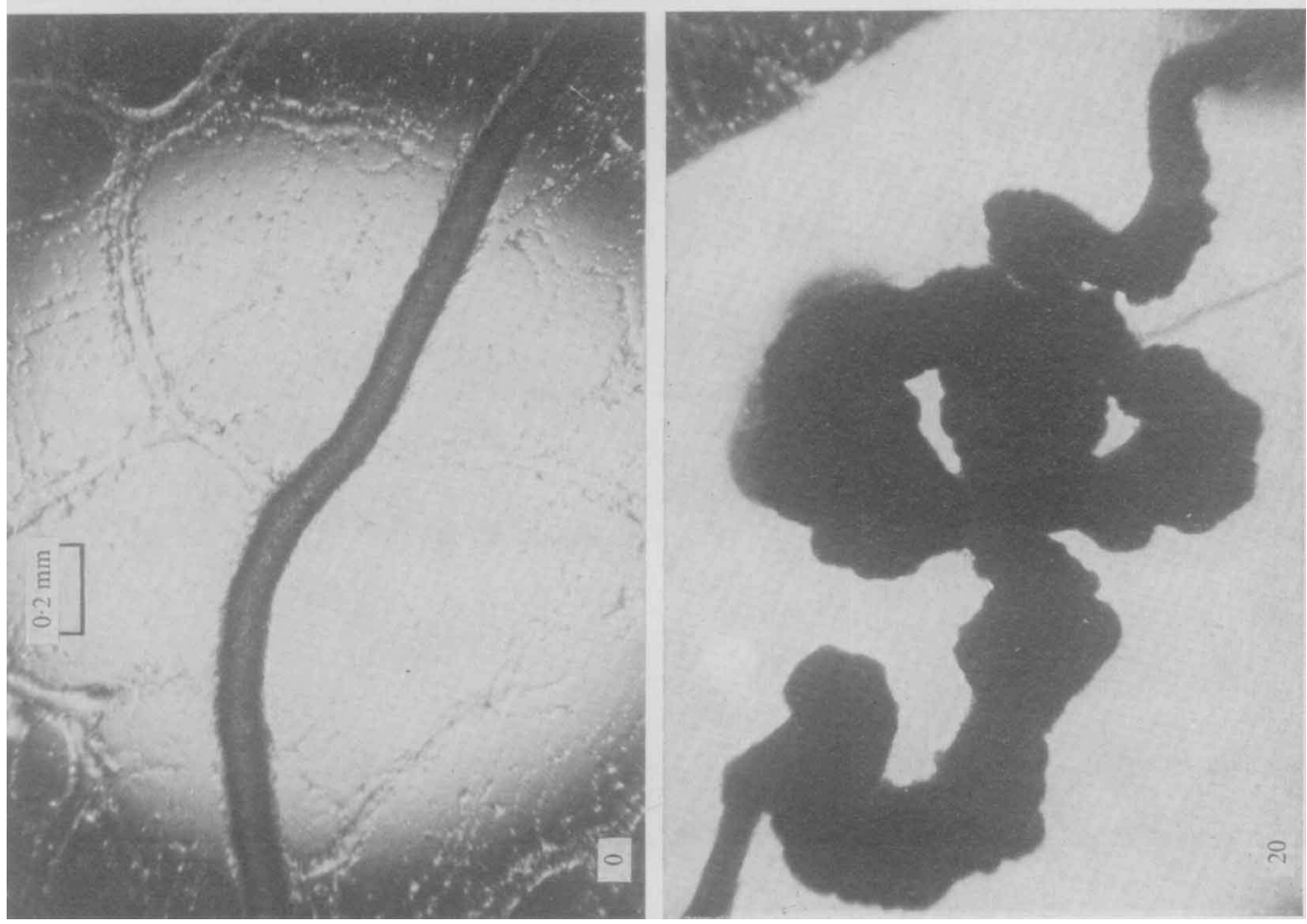

龸若

气 ญ

密気

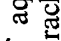

\%

음

๘

등

范

욥

密

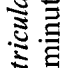

s.

प.

छ․․

응

랭ํㅁ

要

㟔

흘을

悹

宁 产

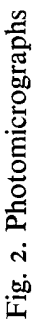


fan towards it. As the plasmodium approached the disc, the precision of its convergence was maintained by the continual withdrawal of lobes at the lateral extremities of the front and the proliferation of others at the part nearest the disc. When the plasmodium touched the $\operatorname{disc}(\mathrm{I} 2 \mathrm{~h}$ I $5 \mathrm{~min}$ ) protoplasm began to flow into the contacting region, leaving the rest much attenuated. The true positive chemotactic response which involved migration then ceased, and became replaced by an internal redistribution of protoplasm through veins of protoplasmic streaming within the plasmodium. The plasmodium later began to migrate away from the disc before all of its protoplasm had accumulated upon it.

Application to plasmodia. Direct application of Stereum extract to plasmodia of Badhamia affected the rhythm of protoplasmic streaming. Normally the endoplasm shuttled back and forth within the veins, the periods of flow in each direction usually lasting 20 to $70 \mathrm{~s}$; the largest and shortest periods of one-way flow observed were $2 \mathrm{~min} 47 \mathrm{~s}$ and $6 \mathrm{~s}$. A pause in the flow, rarely for longer than $5 \mathrm{~s}$, accompanied each reversal. When a minute drop (about $50 \mu \mathrm{l}$ ) of aqueous solution of extract, prepared as described by Madelin et al. (1975), was applied to a single posterior plasmodial strand $0.1 \mathrm{~mm}$ wide, normal behaviour continued for 0.5 to $5 \mathrm{~min}$. The protoplasm then began to flow into the treated area from both directions continuously for a period of 6 to $25 \mathrm{~min}$.

This reversal-suppressing activity of the extract was thermostable, persisting after $20 \mathrm{~min}$ in an autoclave at $115{ }^{\circ} \mathrm{C}$, and migrated in paper chromatograms developed with butanolacetic acid-water with an $R_{F}$ of between 0.83 and 0.96 . In these respects the activity resembled that which produced positive chemotactic responses (Madelin et al. 1975). However the reversal-suppressing activity was rapidly lost on dilution of the preparation. When the standard aqueous preparation was diluted in twofold steps, the period of suppression of reversals dwindled and was not reliably detected beyond eightfold dilution. Presumably the suppression of flow reversals was a consequence of exposure of the plasmodium to very high concentrations of the attractant.

Suppression of flow reversals by direct application of attractant preparations led to the treated strand swelling and becoming highly convoluted (Figs. 2 and 3). The swelling continued even after reversals in protoplasmic flow were resumed. Eventually the entire plasmodium withdrew into a compact mass centered upon the region where the drop was applied. Subsequently it spread more thinly across the agar substrate and resumed normal form 7 to $\mathrm{I} 2 \mathrm{~h}$ after application of the drop. Similar effects were observed when a drop was applied to the anterior margin of a plasmodium.

The morphological consequences of applying drops of $\frac{1}{2}$ and $\frac{1}{4}$ the concentration of the aqueous solution to posterior strands were similar though milder. With $\frac{1}{8}$ concentration, the strand swelled only slightly, rarely to more than twice its original diameter. Greater dilutions caused neither swelling nor convolution of the posterior strand. However, if $\frac{1}{8}, \frac{1}{16}$ or $\frac{1}{3} \frac{1}{2}$ concentrations were applied to a sub-anterior region of a plasmodium there was slight swelling, and from the swollen region branched processes up to $0.4 \mathrm{~mm}$ long extended into the drop and formed lobes, 0.1 to $0.2 \mathrm{~mm}$ diameter, at their apices (Fig. 4). A concentration of $\frac{1}{64}$ was without effect.

\section{The effects of benzamide on plasmodia of Badhamia}

Korohoda, Rakoczy \& Walczak (1969) reported that the direct application of solutions of benzamide $\left(\mathrm{C}_{6} \mathrm{H}_{5} \mathrm{CONH}_{2}\right)$ to plasmodia of three species of myxomycete, including Badhamia utricularis, caused a prolonged cytoplasmic flow into the treated region and accompanying accumulations of cytoplasm. Because this activity resembled that of Stereum extract it was examined.

Application at a distance from plasmodia. Aqueous $0.0 \mathrm{I}, 0.03$ and $0.09 \mathrm{M}$ solutions of 


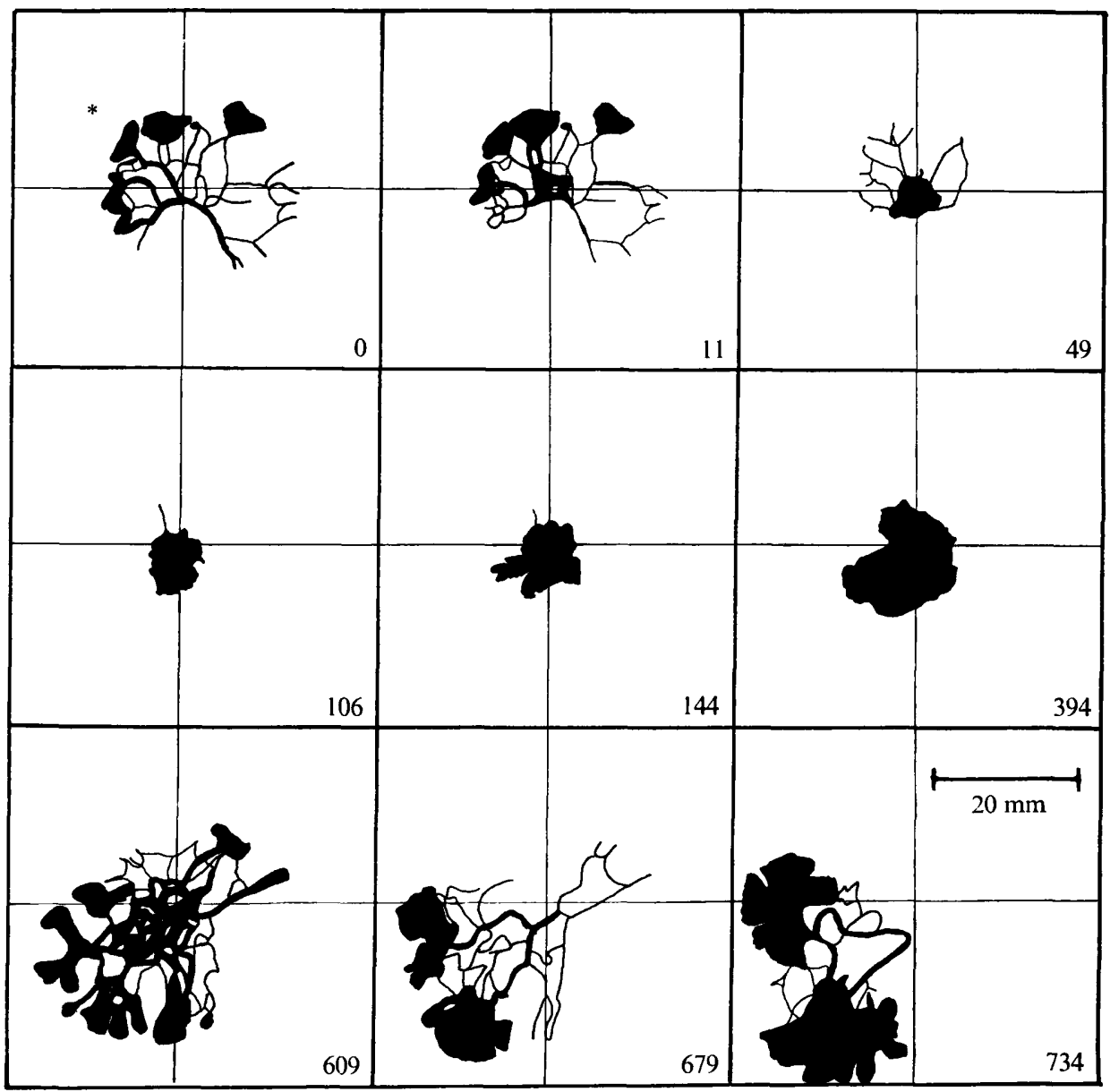

Fig. 3. Tracings of a plasmodium of B. utricularis. A drop of an aqueous solution of Stereum extract was applied to a posterior strand of the plasmodium at the intersection of the crossed lines. Time after application of the drop is given in minutes. The asterisk in the first tracing lies in the direction in which the plasmodium was initially migrating.

benzamide were tested by means of quadrant tests, but no evidence of chemotactic effectiveness was obtained. However, the form of plasmodia altered so that they became single long ( $>60 \mathrm{~mm}$ ), thick $(0.6$ to $0.8 \mathrm{~mm}$ wide) strands, each with a relatively small anterior sheet.

Application to plasmodia. When a minute drop (about $50 \mu \mathrm{l}$ ) of aqueous solution of benzamide at a concentration of $\geqslant 10 \mathrm{mM}$ was applied to a posterior strand of a Badhamia plasmodium, protoplasmic streaming ceased for 5 to $10 \mathrm{~s}$ and then resumed as a rapid centripetal flow from both directions without reversals. The longest period of continuous flow observed was $44 \mathrm{~min}$. Accumulation of protoplasm led to the strand swelling, but not to it becoming convoluted as it did in strands treated with Stereum extract. With benzamide the influx of protoplasm was accommodated in distensions which blew out at many parts of the surface and grew in a discontinuous, jerky manner (Fig. 5). Occasionally, small vesicles ( $0.1 \mathrm{~mm}$ diam) became detached from the rapidly expanding system. The sustained inward flow often continued until the entire plasmodium was concentrated within the treated area, 


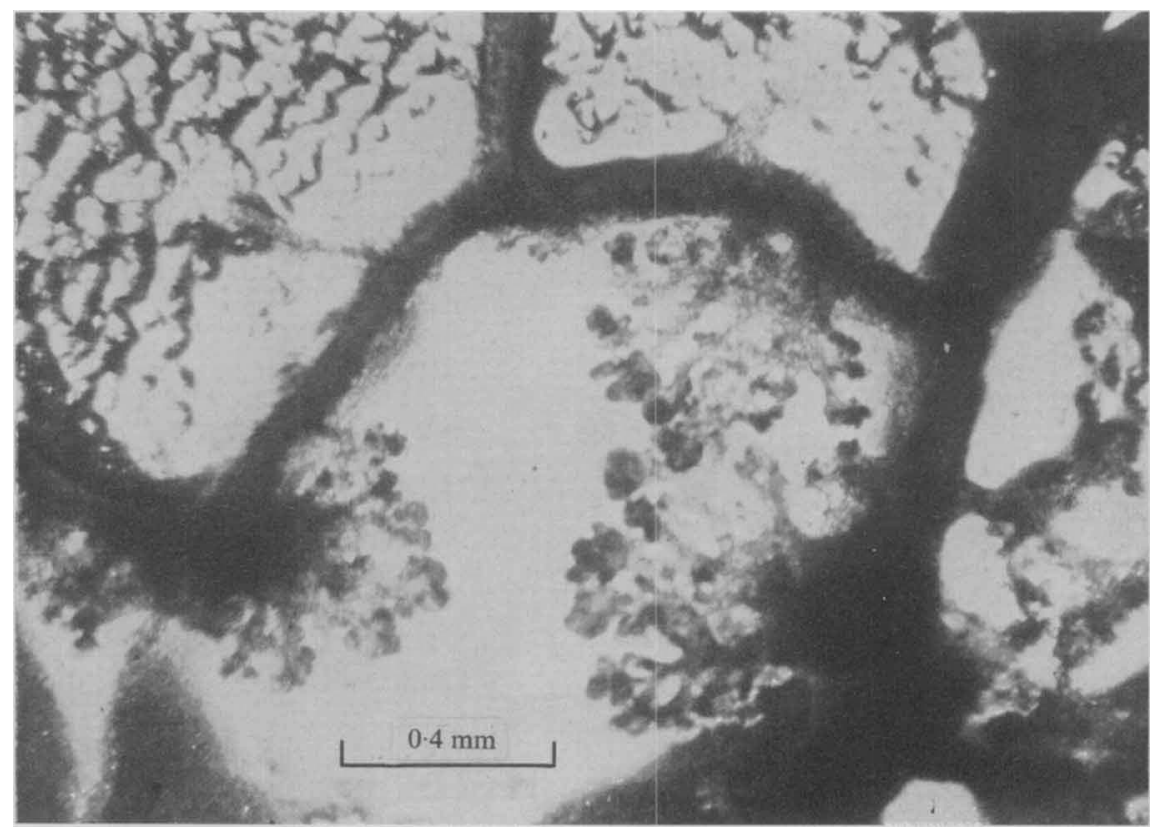

Fig. 4. Photomicrograph of part of the sub-anterior region of a plasmodium of B. utricularis after a drop of diluted $\left(\frac{1}{8}\right)$ aqueous solution of Stereum extract had been applied. Lobed processes have grown out from the strands of plasmodium.

but sometimes reversals of flow recommenced before this happened, general influx of protoplasm ceased and the inflated mass became resorbed into the rest of the plasmodium.

Benzamide thus differed from Stereum extract not only in its lack of positive chemotactic effectiveness but also in respect of significant features of its influence on protoplasmic streaming and accumulation. Firstly, while 10 mM-benzamide applied to plasmodia temporarily halted protoplasmic streaming (an effect reported also by Korohoda et al. 1969), extract of Stereum did not. Secondly, protoplasm accumulated at the site of application of benzamide solution only for as long as one-way protoplasmic flow was sustained. With Stereum extract it accumulated even after resumption of shuttle flow. Thirdly, the influx of protoplasm was accommodated chiefly in laterally blown-out vesicles on the plasmodial strands and not in the greater length of strand, albeit swollen and highly convoluted, which was generated by intercalary extension after treatment with Stereum extract. Fourthly, no tested concentrations of benzamide led to the formation of the small lobed processes (Fig. 4) which arose after treatment with dilute Stereum extract.

\section{The effects of extracts of other fungi on plasmodia of Badhamia}

Whether the chemotactic substance in Stereum extract was species-specific or of widespread occurrence was investigated by studying the effects of extracts of several moulds on plasmodia of Badhamia.

Application at a distance from plasmodia. Extracts of cultures of Metarrhizium anisopliae, Epicoccum nigrum, Helminthosporium dematioideum, Alternaria brassicicola and Penicillium sp. were dried on $5 \mathrm{~mm}$ diameter filter paper discs and tested for chemotactic effectiveness by means of quadrant tests. There was no evidence of chemotactic effectiveness with 


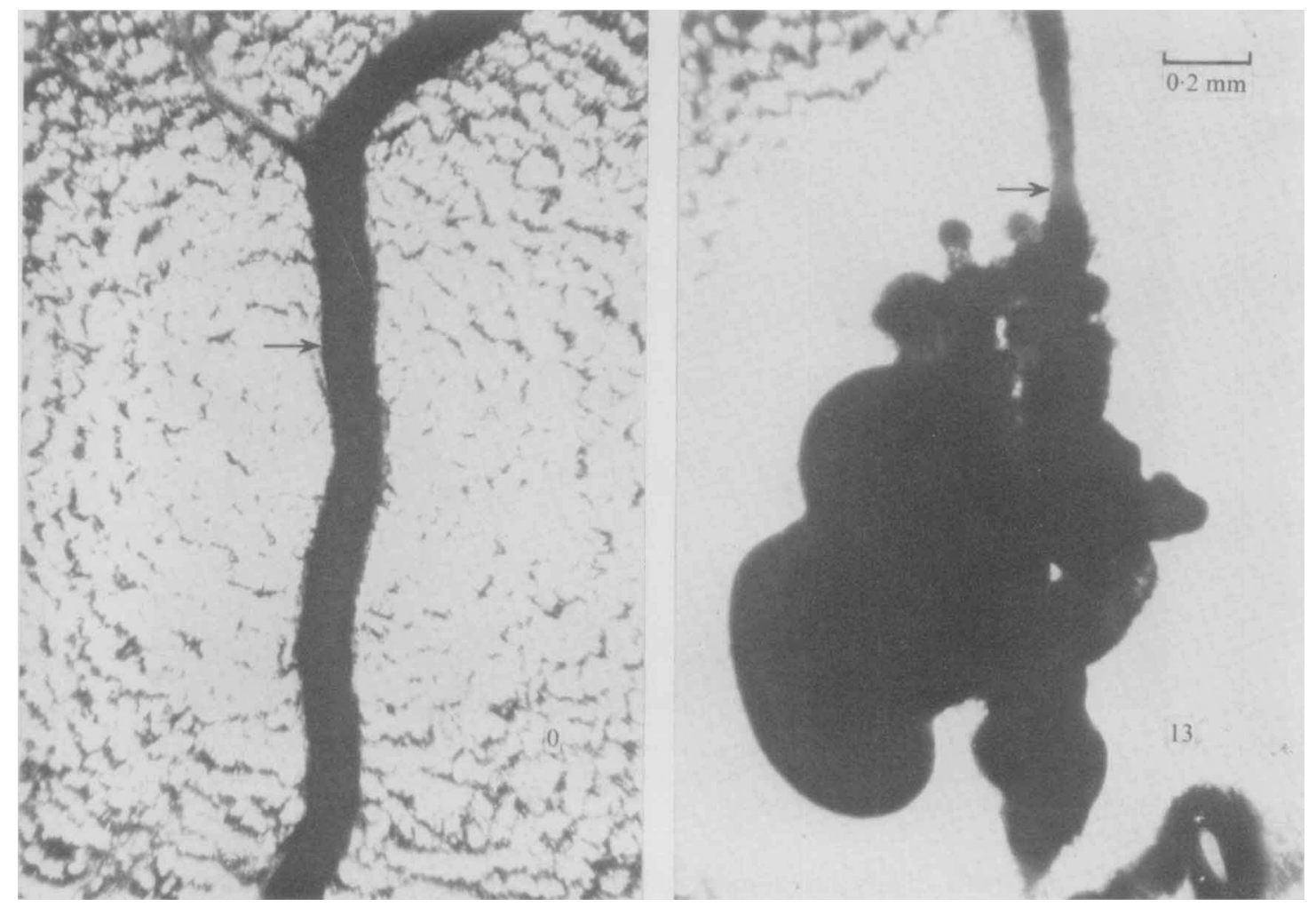

Fig. 5. Photomicrographs of a strand o plasmodium of B. utricularis before and 13 min after the addition of a drop of aqueous $0.05 \mathrm{M}$-benzamide solution. Vesicles have repeatedly blown out from the swelling strand. The differences in background are due to the drop. The arrowed points correspond.

extracts of any species except $M$. anisopliae, which caused all of eight plasmodia to contac . the discs $(P=<0.002 \%)$.

Application to plasmodia. Aqueous derivatives of the five mould species were prepared from standard acetone extracts by the procedure used for $S$. hirsutum extract, and drops (about $50 \mu \mathrm{l}$ ) were applied to various parts of Badhamia plasmodia. Extracts of E. nigrum, $A$. brassicicola and Penicillium sp. elicited no observable responses. Extract of H. dematioideum had no observable effect on protoplasmic streaming but usually caused the treated strand of a plasmodium to become thinner and sometimes to disappear through evacuation of protoplasm. The extract of $M$. anisopliae caused effects identical to those caused by applications of Stereum extract, viz. replacement of reversing protoplasmic streaming by sustained centripetal flow, and the convolution and swelling of the treated strand. The mass of protoplasm which accumulated was usually smaller than with Stereum extract and was later resorbed into the rest of the plasmodium.

Purification of extract of $M$. anisopliae. Acetone extract of colonies of $M$. anisopliae was fractionated by paper chromatography with butanol-acetic acid-water, and longitudinal strips of the chromatogram from origin to solvent front were divided each into 16 equal lengths which were tested eight at a time for chemotactic effectiveness in clock tests. Plas- 
modia migrated to pieces of chromatogram corresponding to an $R_{F}$ of 0.88 to I $\circ$. A concurrently prepared, identically treated chromatogram of Stereum extract revealed chemoattractant activity at $R_{F} 0.83$ to 0.96 , which within the limits of resolution is the same range as previously reported by Madelin et al. (1975).

The effects of direct application of drops of eluates from the chromatogram were determined. Eluates corresponding to $R_{F} 0.88$ to $\mathrm{I} \cdot 0$ caused a slight swelling when applied to solitary posterior strands of Badhamia plasmodium, but when placed on the sub-anterior zones of plasmodia they caused small lobed processes to form on the surfaces of strands in contact with the drops. These were identical to the ones formed after similar applications of low concentrations of Stereum extract (Fig. 4). Eluates of other regions of the chromatogram were without observable effect.

\section{DISCUSSION}

The extracts of $S$. hirsutum and $M$. anisopliae were chemotactically effective if presented at a distance from the Badhamia plasmodium, and suppressed reversals of protoplasmic streaming and caused swelling if applied directly. These effects appear to be related and to be caused by a single agent acting at different concentrations. This is inferred from the following considerations. The locomotion of the slime-mould plasmodium is closely connected with protoplasmic streaming, without which it cannot take place. Such streaming proceeds back and forth alternately. The difference between the volumes of protoplasm carried in opposite directions contributes to the locomotion of the plasmodium, which advances in the direction in which the larger volume is transported (Kamiya, I959). Kamiya (1950) demonstrated such differences between the volumes of protoplasm transported in forward and reverse directions, and correlated these with the direction of movement of the plasmodium. An exogenous substance need only maintain this asymmetry in volume transported to be chemotactically effective. The mode of orientation of the migrating plasmodium of Badhamia towards the source of attractant by means of the formation of new lobes at the margin nearest the source and the withdrawal of those furthest from it (Fig. I) is compatible with the operation of such a mechanism. At higher concentrations such an exogenous substance might so exaggerate this asymmetry that reversed flow is entirely suppressed. Unidirectional flow, and consequent vigorous accumulation of protoplasm in the treated strands, could therefore be an extreme response in a series which, at lower concentrations of the exogenous agent, includes positive chemotaxis. The accumulation of protoplasm in strands to which extract was applied, and which continued even after reversals in flow had been resumed, was presumably a consequence of persistent asymmetry in volume transported back and forth. Every gradation of response between completely symmetrical protoplasmic transport and unidirectional streaming is possible; present evidence suggests that the degree of asymmetry reflects the concentration at which the active extract is presented to the plasmodium.

The substance in $S$. hirsutum which causes chemotaxis is probably a secondary metabolite, and one which at least one other fungus ( $M$. anisopliae) produces. Metarrhizium anisopliae is known to produce cytochalasins C and D (Aldridge \& Turner, 1969) and other secondary metabolites (Kodaira, I96I; Turner, I97I), but the effects of these on Badhamia have not been investigated.

Because the extract of Stereum hirsutum partially resembles benzamide in its activity, it may share some of benzamide's modes of action. Korohoda et al. (I969) believed that this non-volatile anaesthetic disturbed protoplasmic streaming patterns by acting on the plasma membrane which, they argued, controlled the ectoplasmic contractions responsible for 
bulk streaming of endoplasm. Action on the plasma membrane is a possible explanation of the activity of Stereum extract, particularly in view of its capacity for inducing curiously modified configurations of the plasmodial surface (Fig. 4) and for stimulating massive yet orderly increases in observable surface area of the plasma membrane (Fig. 2). WohlfarthBotterman (I974) has shown that, as a result of deep and complex invaginations, strands of the plasmodium of Physarum polycephalum have a plasmalemma surface area two- to tenfold larger than their apparent surface area. Whether the great increase of surface area in Badhamia plasmodia reflects new synthesis of membrane or extension of a highly convoluted surface, or both, remains to be determined. The capacity to induce large increases in the surface area of plasmodia of Badhamia is a property of benzamide also (Fig. 5; and Korohoda et al. 1969), but it is accompanied by uncontrolled blebbing. Stereum extract is also different from benzamide in its vigorous positive chemotactic effectiveness. Significant differences between their modes of action are evident despite some similarities.

\section{REFERENCES}

Aldridge, D. C. \& Turner, W. B. (1969). Structures of cytochalasins C and D. Journal of the Chemical Society, Section C. Organic Chemistry 1969, 923-928.

KamiYA, N. (1950). The protoplasmic flow in the myxomycete plasmodium as revealed by a volumetric analysis. Protoplasma 39, 344-357.

KamiYa, N. (1959). Protoplasmic streaming. Protoplasmatologia 8, I-I 99.

KodaIRA, Y. (196I). Biochemical studies on the muscardine fungi in the silkworms, Bombyx mori. Journal of the Faculty of Textile Science and Technology, Shinshu University, Series E, Agriculture and Sericulture 5 , I-68.

KoROHODA, W., RAKOCZY, L. \& WALCZAK, T. (1969). Effects of benzamide upon protoplasmic streamings and electric activity in slime molds plasmodia. Folia biologica (Praha) 17, 195-209.

Madelin, M. F., Audus, F. \& KNowles, D. (1975). Attraction of plasmodia of the myxomycete, Badhamia utricularis, by extracts of the basidiomycete, Stereum hirsutum. Journal of General Microbiology 89, 229-234.

Turner, W. B. (1971). Fungal Metabolites. London and New York: Academic Press.

Wohlfarth-Botterman, K. E. (1974). Plasmalemma invaginations as characteristic constituents of plasmodia of Physarum polycephalum. Journal of Cell Science 16, 23-37. 